\title{
Die Wirkung von psychischem Streß auf das Immunsystem. Ein weiterer Grund für tiergerechte Haltung (Übersichtsreferat)
}

\begin{abstract}
Summary
Title of the paper: The effect of psycho stress on the immune system. Another reason for pursuing animal welfare (Review)

Stress can be seen as the body's most important and complex reaction to ensure survival. Thus, stress must be considered a fundamentally positive type of adaptive reaction and concepts of stress have to be integral parts in considering animal well-being.

It is widely recognized that acute and chronic stress have an impact on the neuroendocrine and immune system, the latter being of special interest with respect to health and welfare of animals.

This review intends to provide an integrative approach to the complex relationships between stress, behaviour, neuroendocrine and immune system of farm animals. Physiological mechanisms that mediate the effects of stress on immune function including basic mechanisms of neuroendocrine-immune network and principles of immunomodulation are presented and discussed in consideration of their practical impact for livestock production.
\end{abstract}

Key Words: stress, immune system, immunomodulation, neuroendocrine-immune network, farm animals

\section{Zusammenfassung}

Streß ist als nichtspezifische Antwort des Organismus auf beliebige Reize hin definiert. Dabei handelt es sich um differenzierte Anpassungsmechanismen zur Aufrechterhaltung der Homøostase, die neben neuroendokrinen Reaktionen auch Funktionen des Immunsystems beeinflussen. Diese Effekte auf das Immunsystem gewinnen im Zusammenhang mit tiergerechter Haltung, Gesundheit und Wohlbefinden landwirtschaftlicher Nutztiere zunehmend an Bedeutung.

Es werden der derzeitige Kenntnisstand der Zusammenhănge zwischen Streß und Immunităt bei landwirtschaftlichen Nutztieren einschließlich der zu Grunde liegenden physiologischen Mechanismen dargestellt und praktische Konsequenzen fur die Nutztierhaltung abgeleitet.

Schlüsselwörter: Streß, Immunsystem, Neuroendokrinum, Regulation, Netzwerk, landwirschaftliche Nutztiere

1. Streß und Immunsystem

1.1 Streß

Streß im weitesten Sinne ist ein Prozeß der Anpassung der psychophysischen Ressourcen eines Individuums an die Anforderungen, die die Umwelt an es stellt (SCHEDLOWSKI und SCHMIDT, 1996). Wenn sich das homöostatische Gleichgewicht im Organismus nicht durch unwillkürliche Mobilisierung von Ressourcen oder durch reflexartige Verhaltensänderung wiederherstellen läßt, reagiert der Organismus mit einer Alarmreaktion.

Streß ist demnach die unspezifische Antwort des Organismus auf unerwartete und auf irgend eine Weise bedeutsame Reize. Die Streßreaktion soll helfen, bedrohliche und belastende Situationen mittels Veränderungen des physiologischen Zustands sowie des Verhaltens zu meistern. Dabei sind Psyche und Körper beteiligt. Streß kann sich so- 
wohl positiv als auch negativ auf ein Individuum auswirken. Schädlich wird er besonders dann, wenn er unvorhersehbar und unkontrollierbar wird.

Stressoren lassen sich nicht nur nach Art und Intensität, sondern auch nach Dauer und Häufigkeit unterscheiden. In der Literatur gibt es keine eindeutigen Kriterien dafür, bis wann ein Streß noch als akut und ab wann er als chronisch klassifiziert werden kann. Sinnvoll erscheint es, solche Reaktionen als akut zu bezeichnen, bei denen es kurzfristig im inneren Milieu zu einer Abweichung des Istwertes vom Sollwert kommt und sich anschließend relativ schnell der Ausgangswert wieder einstellt. Als chronischen Streß könnte man dann eine Reaktion beschreiben, bei der es zu anhaltender Erhöhung des Istwertes für streßrelevante biologische Systeme, beispielsweise Hormone, kommt. Ob ein Reiz die Funktion eines Stressors hat und ob der Stressor akut oder chronisch ist, läßt sich letztendlich nur an der Reaktion des Organismus erkennen (TEWES, 1996). Als dritte Streßkategorie wurde der chronisch intermittierende Streß vorgeschlagen (BURCHFIELD, 1979). Hier wirkt ein akuter Stressor ein, der in regelmäßigen oder unregelmäßigen Abständen wiederholt wird.

In einer Vielzahl von Untersuchungen konnte gezeigt werden, daß Streßeinwirkungen nicht nur neuroendokrine Reaktionen, sondern auch Funktionen des Immunsystems auf zellulärer und humoraler Ebene beeinflussen können. Diese Effekte auf das Immunsystem gewinnen im Zusammenhang mit tiergerechter Haltung und Gesundheit landwirtschaftlicher Nutztiere zunehmend an Bedeutung.

\subsection{Streßreaktionen}

Bei der zentralnervösen Verarbeitung von psychischem Streß kommt es zu einer Erregung von Teilen des limbischen Systems, die nicht nur zur Aktivierung des Hypophysenvorderlappens mit nachfolgender Ausschüttung von adrenocorticotropem Hormon (ACTH) führt, das wiederum die Nebennierenrinde zur Glucocorticoidproduktion veranlaßt (HPA-System: Hypophysis-Pitutary-Adrenal Cortex), sondern gleichzeitig auch über den Nukleus coeruleus und den Hypothalamus eine Sympathikuserregung bewirkt (SAM-System: Sympathicus-Adrenal Medulla). Während das Nebennierenmark vermehrt Adrenalin produziert, wird aus den Nervenendigungen des Sympathikus vor allem Noradrenalin ausgeschüttet. Durch diese Katecholamine werden Belastbarkeit und Leistungsfähigkeit des Organismus bei Kampf- und Fluchtreaktionen erhöht (Verbesserung der Durchblutung von Herz- und Skelettmuskulatur, Erhöhung der Herzschlagfrequenz, vermehrte Energiebereitstellung durch Erhöhung von Lipolyse und Proteolyse). Die zwei Komponenten, HPA- und SAM-System, werden von CHROUSOS und GOLD (1992) als bedeutendste Vermittler der Streßantwort angesehen.

\section{Kontrollerwartung und Kontrollmöglichkeit, Vorhersehbarkeit}

Versuchstiere, die nach einem Zufallsschema stark aversiven Reizen (Elektroschocks) ausgesetzt werden, zeigen physiologisch ausgeprägtere Streßreaktionen als jene Tiere, die gleich vielen und gleich intensiven aversiven Reizen ausgesetzt werden, diese jedoch als Konsequenz des eigenen Verhaltens wahrnehmen (Bestrafung) (TEWES, 1996). Die subjektive Belastung bei stark aversiven Reizen wird bei Kontrollgewißheit 
und vorhandener Kontrollmöglichkeit stark vermindert.

Die Kontrollerwartung wird wiederum durch die persönliche Lerngeschichte beeinflußt. Wenn ein Individuum auf Dauer die Erfahrung macht, daß die Konsequenzen des eigenen Verhaltens völlig unvorhersehbar sind, kann seine Kontrollerwartung stark gemindert sein. Das subjektive Gefuhl der Hilflosigkeit wird dann generalisiert, obwohl objektiv noch Möglichkeiten der Reiz- bzw. Situationskontrolle bestehen können. Man spricht in diesem Falle von einer „erlernten Hilflosigkeit" (SELIGMAN und BEAGLEY, 1975).

\section{$1.4 \quad$ Psychoneuroimmunologie}

Lange Zeit wurden Nerven- und Immunsystem als weitgehend voneinander unabhăngig funktionierend angesehen. Mit den Experimenten zur konditionierten Immunsuppression bei Ratten lieferten ADER und COHEN (1975) den klassischen Beweis für die Existenz von Wechselwirkungen zwischen Emotionen und ihren zentralnervösen Repräsentationen einerseits und dem Immunsystem andererseits. In den Versuchen wurde den Tieren eine Saccharinlösung gepaart mit immunsuppressivem Cyclophosphamid verabreicht. Nach dieser kombinierten Gabe löste Saccharin alleine immunsuppressive Wirkungen aus.

$\mathrm{Da}$ psychische Prozesse das Immunsystem auch beim Menschen beeinflussen können, zeigten die Studien zu Prüungsbelastungen von KIECOLT-GLASER et al. (1984). Die Schwächung des Immunsystems erhöhte das Risiko einer Infektionskrankheit vor allem bei den Studenten, bei denen der Prüungsstreß mit Mangel an sozialen Kontakten verbunden war.

Seitdem belegen die Ergebnisse zahlreicher Untersuchungen, daß Nerven-, Hormonund Immunsystem in einem biochemischen Netzwerk miteinander kommunizieren (Übersichten bei MADDEN und FELTEN, 1995; OTTAVIANI und FRANCESCHI, 1996; McEWEN et al., 1997; VON HOLST, 1998). Eine neue Forschungsrichtung, häufig als "Psychoneuroimmunologie" bezeichnet, entstand mit dem Ziel, immunmodulatorische Eigenschaften von Streß aufzudecken und deren potentielle Bedeutung für die Gesundheit abzuschätzen.

Regulatorische Impulse wirken nicht nur vom Zentralnervensystem (ZNS) auf das Immunsystem ein, Informationen gelangen auch umgekehrt vom Immunsystem zum ZNS. Die morphologische Grundlage furr diese Interaktionen ist mit der Innervation der primären (Knochenmark, Thymus) und sekundären (Lymphknoten, Milz) Organe des Immunsystems durch noradrenerge Nervenfasern, die aus den Ganglien des sympathischen Nervensystems entspringen, gegeben (FELTEN et al., 1985). Neben dieser direkten Innervierung schafft die Existenz von Rezeptoren für Neuropeptide, Neurotransmitter und Hormone auf Zellen des Immunsystems eine biochemische Verbindung für eine zentralnervöse Immunmodulation. Auf immunkompetenten Zellen konnten beta-adrenerge, dopaminerge, cholinerge und verschiedene Opioidrezeptoren sowie Rezeptoren für Somatostatin, Vasopressin, Prolaktin, Substanz P und Neuropeptid Y nachgewiesen werden (STEFANO, 1989; ADER et al., 1990; SCHLENKER und LYHS, 1991; LANDMANN, 1992; PELLEGRINI et al., 1992; ATHREYA, 1993). Das aktivierte Immunsystem kann wahrscheinlich die meisten dieser neuroendokrinen Hormone selbst produzieren (STEPHANOU et al., 
1990; BLALOCK, 1994).

Von zentraler Bedeutung für die Informationsübertragungen zwischen Immunsystem und zentralem Nervensystem sind die von aktivierten Monozyten und Makrophagen gebildeten Zytokine Interleukin-1beta (IL-1 $\beta$ ), Interleukin-6 (IL-6) und Tumornekrosefaktor-alpha (TNF- $\alpha$ ), wobei das System von Interleukin-1 derzeitig am besten erforscht ist. IL-1 kann sowohl indirekt über Endothelzellen der Gehirngefäße als auch direkt über bestimmte Nervenbahnen (Vagus) auf Nervenzellen im Gehirn Einfluß nehmen. Interieukine können aber auch in Mikrogliazellen, Astrozyten und Neuronen selbst gebildet werden (Übersicht bei FABRY et al., 1994).

Das limbische System wird vor allem als Integrationsebene im Zusammenwirken mit dem HPA-System betrachtet. Homöostaseafferenzen des Körperinnern und vorverarbeitete sensorische Signale aus der Umwelt werden hier zusammengeführt und wenn möglich mit Erfahrungen verglichen, um eine adäquate Aktivität auszulösen (OTTAVIANI und FRANCESCHI, 1996).

Eine Schlüsselsubstanz ist dabei das Corticotropin-Releasing-Hormon (CRH), das im Hypothalamus und anderen limbischen Hirngebieten produziert wird und gleichsinnigen Verlauf von Streß- und Immunreaktion bewirkt (JOHNSON et al., 1994; KARALIS et al., 1997). Rezeptoren für CRH wurden außerhalb des Gehirns insbesondere auf Makrophagen der Milz bei Ratten und Mäusen identifiziert (WEIGENT und BLALOCK, 1995; WEBSTER et al., 1997).

Die Komplexität des Systems begründet die Schwierigkeit, immuno-neuroendokrine Interaktionen vollständig zu analysieren. Das Gesamtsystem stellt sich als dynamisch rückgekoppeltes Netzwerk dar, das in seinen Reaktionen kritisch von subtilen zeitlichen quantitativen Zuständen und Wechselwirkungen seiner Elemente abhängt (Abb. 1).

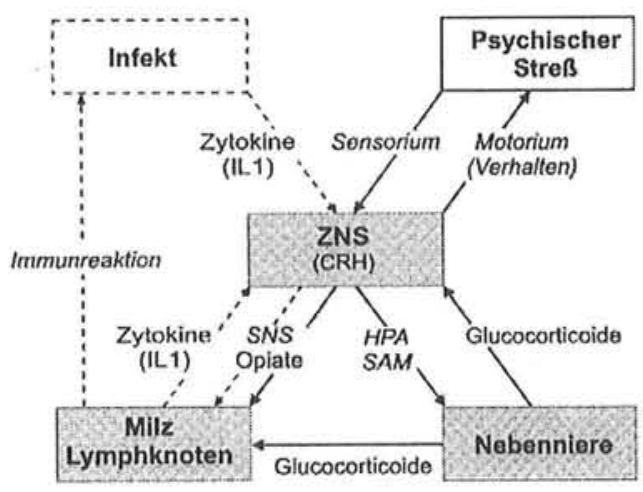

CRH: Corticotropin Releasing Hormon

IL: Interleukin

SNS: Sympathisches Nervensystem

HPA: Hypothalamo-hypophysär-adrenales System

SAM: Sympatho-adrenomedullăres System

Abb. 1: Schematische Darstellung der für die Streßreaktion bedeutsamen Wechselwirkungen zwischen Zentralnervensystem (ZNS), Hormon- und Immunsystem (Scheme illustrating the neuroendocrine-immune system interactions)

\subsection{Streßeffekte auf Immunfunktionen}

In der Literatur wurden unterschiedlichste Wirkungen verschiedener Stressoren auf Immunfunktionen berichtet. Die vereinfachte Einteilung, daß kurzfristige Belastungen 
generell eine Steigerung immunologischer Funktionen bewirken, länger andauernde Belastungen hingegen Immunfunktionen hemmen, ist allenfalls als grobe Faustregel haltbar.

Es gibt kaum ein Hormon, das nicht in seiner Wirkung auf das Immunsystem untersucht wurde (COMSA et al., 1982), wobei widersprüchliche Ergebnisse bestehen. So wird bei Glucocorticoiden, $\mathrm{CRH}, \mathrm{ACTH}$, Androgenen, Progesteron und Katecholaminen ein hemmender Effekt, hingegen bei Prolaktin, Insulin, Thyroxin, Wachstumshormon, beta-Endorphin und Enkephalinen ein stimulierender Effekt auf Funktionen des Immunsystems angenommen. Für eine optimale Funktion der Zellen des Immunsystems konnte die Notwendigkeit eines ausgewogenen Verhältnisses von katabolen (Glucocorticoide) und anabolen Hormonen (Insulin, Somatotropes Hormon) nachgewiesen werden (Zweikomponententheorie von SCHOLE, 1982).

Antigene Stimuli lösen die Kommunikation vom Immun- zum Zentralnervensystem durch Freisetzung von Zytokinen (IL-1, TNF- $\alpha$ ) durch aktivierte Monozyten und Makrophagen aus, so daß das Immunsystem über diese afferenten Signale auch die HPA-Achse regulieren kann.

Inzwischen häufen sich Hinweise, daß nicht nur psychische Faktoren die Immunfunktion modulieren (z.B. durch Konditionierung), sondern daß auch die Immunfunktion das Verhalten beeinflussen kann (DANTZER et al., 1993). Die intrazerebroventrikuläre Injektion von IL-1 oder TNF- $\alpha$ bzw. Endotoxingabe, die die IL-1-Produktion in Makrophagen induziert, provoziert unspezifische Krankheitssymptome mit stresscharakteristischen Verhaltensweisen (Krankheitsverhalten, „sickness behaviour"). Zur Beeinflussung des Nahrungsaufnahmeverhaltens durch IL-1 und TNF- $\alpha$ liegen Untersuchungen beim Schwein von JOHNSON und VON BORELL (1994) sowie WARREN et al. (1997) vor. Endotoxinbehandlung erhöht Immobilität und Schläfrigkeit und vermindert das soziale Explorationsverhalten. Die Ergebnisse verdeutlichen, daß IL-1 der Hauptfaktor bei der Modulation der sozialen Komponenten des „sickness behaviour" zu sein scheint (KENT et al., 1992). Andererseits zeigen Ergebnisse an Ratten, daß $\mathrm{CRH}$ wiederum die Verhaltenseffekte von IL-1 modulieren kann (BLUTHE et al., 1992).

Streßassoziierte Immunsuppression wird primär über die HPA-Achse vermittelt. Diese Verbindung erklärt jedoch nicht alle immunhemmenden Streßeffekte und vor allem nicht den immunstimulierenden Effekt von positivem Streß (Eustreß). Von einigen Autoren wird die Opioidstreß-Hypothese zur Erklärung von Immunmodulation durch Streß favorisiert, da diese die Einbeziehung der psychologischen Qualität kognitiver Stimuli erlaubt (STEFANO, 1989).

Die Dimension von Ausweglosigkeit/Kontrollierbarkeit bzw. Unsicherheit/Sicherheit der Psychostressoren wird zwar von der Situation ausgelöst, aber nicht definiert. Die Beherrschbarkeit, das Bewältigen einer Situation, wird von der individuellen Einschätzung der Situation und dem instinktiven oder kognitiven Reaktionsschema bestimmt. Empfindung, Interpretation, Abschätzung lassen Distre $ß$ oder Eustre $ß$ entstehen. Die Konditionierung emotionaler/psychologischer Streßdimensionen kann die Reaktion bei wiederholter Exposition gegenüber dem Stimulus lebenslang fixieren. SELYE (1979) spricht von der Selbstregulation der Streßantwort. Wegen der Individualität und Situationsabhängigkeit von $S$ treß sind Differenzen in der Streßperzeption von verschiede- 
nen Spezies zu berücksichtigen. Wesentlich ist demzufolge auch, daß speziesrelevante Stressoren in Experimenten eingesetzt werden.

Bei der immunstimulierenden Wirkung von psychosozialen Faktoren handelt es sich vorrangig um die Stimulierung unspezifischer Parameter des Immunsystems. Die Stimulierung der Natürlichen Killerzell (NK)-Aktivität, der T-Lymphozytenreaktion nach Mitogeninduktion, der Anstieg zirkulierender T-Helferzellzahlen, Absinken der Anzahl zirkulierender zytotoxischer Suppressorzahlen und Zunahme der spontanen lymphozytären Blastogenese wurden wiederholt nachgewiesen (KOLB und REINHARDT, 1990; RUNGE, 1991; MAYR und MAYR, 1998).

Ein Anstieg von Antikörpertitern für verschiedene Viren kann durch psychologische Stressoren bedingt sein und gilt als Ausdruck der psychogenen Aktivierung einer latenten Infektion (GLASER et al., 1985). Harmlose Infektionen durch opportunistische Keime können leichter in Krankheiten konvertieren. Die Tiere sind anfälliger, wodurch besonders die Entstehung von infektiösen Faktorenkrankheiten begünstigt wird.

Streßeffekte auf immunologische Funktionen beim landwirtschaftlichen Nutztier

Bei landwirtschaftlichen Nutztieren konzentrierte sich die Forschung in den letzten Jahren besonders auf die Effekte von unterschiedlichen Haltungs- und Umweltbedingungen auf Immun- und Regulationsfunktionen. Das Mischen unbekannter Tiergruppen, der Transport von Tieren, ihre soziale Isolation sowie Kälte- und Hitzestreß sind wiederholt auftretende potenzielle Streßsituationen, die auch in Belastungsexperimenten untersucht wurden.

Die Experimente mit landwirtschaftlichen Nutztieren haben sich bisher auf spezifische Immunparameter ( $\mathrm{T}$ - und B-Zellproliferationsantworten, Antikörpersynthese, sekretorisches IgA, quantitative Bestimmung der T-Zellsubpopulationen) konzentriert. Häufig kam man zu widersprüchlichen Ergebnissen oder es konnten keine signifikanten Beziehungen zwischen Streßfaktoren und Immunparametern festgestellt werden. Gründe dafür könnten sein, daß tatsächlich keine Beziehungen bestehen oder daß das Zeitregime und der gewählte Immunparameter nicht zueinander passen. Die streßinduzierten immunmodulatorischen Effekte sind wie auch andere Meßwerte eine Funktion der Zeit. Außerdem scheinen Qualität und Dauer von Streßeinwirkungen und die Ausprägung adäquater Bewältigungsstrategien einen wesentlichen Einfluß auf die homöostatischen Regelmechanismen von Immunfunktionen zu nehmen.

Im Einzelnen ergab sich für die wichtigsten Arten landwirtschaftlicher Nutztiere folgendes Bild:

\section{$2.1 \quad$ Schaf}

Isolationsstreß mit zusätzlicher Bewegungseinschränkung führte bei Schaflämmern zu signifikanten Verringerungen der Lymphozytenproliferationsfähigkeit nach unterschiedlicher Mitogeninduktion, die sich jedoch nicht allein mit Hilfe der gemessenen Cortisolwerte erklären ließen (MINTON et al., 1995). In den Isolationsexperimenten von COCKRAM et al. (1994) wurden bei den Lämmern Umverteilungen innerhalb der 
Lymphozytensubpopulationen (erhöhte Neutrophilenzahl, verminderte CD2- und T19Lymphozytenzahl) nachgewiesen.

Schafe, die hohen Umgebungstemperaturen $\left(35^{\circ} \mathrm{C}\right)$ ausgesetzt wurden, reagierten darauf mit einer Verminderung der Lymphozytenreaktivität (NIWANO et al., 1990).

Sowohl Parameter der humoralen Immunantwort als auch Plasmacortisolkonzentrationen von Schaflämmern unterschieden sich nicht zwischen abruptem und partiellem Absetzen vom Muttertier (ORGEUR et al., 1998). In den Untersuchungen von RHIND et al. (1998) führte das Absetzen zwar zu einem Cortisolanstieg bei den Lämmern, humorale und zellvermittelte Immunantworten wurden dadurch jedoch nicht gehemmt.

\section{$2.2 \quad$ Rind}

Transportstreß führte bei Kälbern zu einer verminderten Immunreaktivität, besonders in Bezug auf eine starke Verringerung von IL-2 Rezeptoren auf peripheren Lymphozyten (LAN et al., 1995). Ähnliches fand man bei Bullen (KEGLEY et al., 1997). Darüber hinaus wird ein lymphokinähnlicher Faktor diskutiert, der suppressive Effekte von Glucocorticoiden auf T-Helfer-Zellfunktionen blocken kann. Aber auch Prolaktin ist in der Lage, die Immunsuppression durch Glucocorticoide aufzuheben (BISWAS et al., 1992; MATERA et al., 1992; LÖHRKE et al., 1994).

Die agonistischen Auseinandersetzungen nach dem Mischen von Fleischrindern in kleinen Gruppen (Gruppengröße 10) führte bei sozial subordinaten Tieren nur zu einer tendenziellen Cortisolerhöhung, die keine wesentlichen Veränderungen der Leukozytenzahl und des Verhältnisses der Anzahl von Neutrophilen und Lymphozyten zur Folge hatte (MENCH et al., 1990). Offensichtlich war der Platz für eine Gruppe von 10 Tieren ausreichend. Bei Kälbern hingegen war ein verringertes Platzangebot mit einer signifikanten Reduzierung der T-Lymphozytenproliferation verbunden (FERRANTE et al., 1998). Der Lymphozytentransformationstest wird als Streßindikator empfohlen.

\subsection{Schwein}

McGLONE et al. (1993) beobachteten bei Schweinen mit niedrigem bzw. intermediärem Sozialstatus nach einem 4-stündigen Transportstreß eine signifikant verringerte NK-Zytotoxizität, die wiederum das Infektionsrisiko dieser Tiere erhöhen kann. Mit Hilfe der Plasmacortisolwerte konnten die Belastungseffekte auf die NKAktivität nicht erklärt werden, da die Tiere mit hohen Cortisolwerten (HPAAktivierung) auch eine gesteigerte zytotoxische Aktivität der NK-Zellen aufwiesen. Diese erhöhte NK-Aktivität bei gestreßten Tieren könnte durch Katecholamine, ACTH oder $\beta$-Endorphin vermittelt werden. Der Transportstreß führte außerdem zum signifikanten Anstieg der Neutrophilen und zum Abfall der Lymphozyten.

Während eines einstündigen Transportes von Jungsauen zeigte sich ein wesentlicher Anstieg der Plasmakonzentrationen von Adrenalin und Cortisol sowie ein tendenzieller Anstieg des Cortisol bindenden Proteins (CBG). Gleichzeitig erhöhte sich die Leukozytenzahl im peripheren Blut, wobei die Anzahl der Lymphozyten fiel, die der Neutrophilen stieg an. Die Lymphozytenproliferationsrate nach Mitogenstimulation blieb von der Transportbelastung trotz erhöhter Cortisolwerte unbeeinflußt. Dieses Er- 
gebnis unterstützt die von YANG und SCHULTZ (1986) diskutierte Steroidresistenz der Lymphozyten adulter Schweine. Ebenso erhöhte eine periphere Gabe von Cortisol oder ACTH zwar die Plasmacortisolkonzentrationen, blieb jedoch ohne signifikanten Einfluß auf die NK-Zytotoxizität bei Mastferkeln (SALAK-JOHNSON et al., 1996).

Die gleichzeitige Aktivierung der HPA- und SAM-Achse während des Transportes von Schweinen sehen DALIN et al. (1993) als Hauptergebnis ihrer Untersuchungen an. Hingegen führten Untersuchungen zum Sozialstatus bei Mastferkeln im Zusammenhang mit verschiedenen akuten Belastungssituationen (Transport, Hitze bzw. Kälte) zu unterschiedlichsten Veränderungen der Plasmacortisolwerte und der gemessenen Immunparameter (HICKS et al., 1998). Nur die Verhaltensänderungen waren ein beständiger und zuverlässiger Streßindikator.

DEGUCHI und AKUZAWA (1998) fanden 1h nach dem Mischen von unbekannten 64 Tage alten Mastferkeln signifikant erhöhte Plasmacortisolwerte im Vergleich zu den Werten $1 \mathrm{~h}$ vor dem Mischen und $24 \mathrm{~h}$ danach. Die mitogeninduzierten Lymphozytenproliferationsraten waren jedoch erst 3d nach dem Mischen im Vergleich zu unbelasteten Kontrolltieren signifikant reduziert. Diese signifikante Verringerung der immunologischen Reaktionsfähigkeit blieb bis 19d nach der Gruppenzusammenstellung erhalten. Die streßinduzierten Effekte auf Hormonspiegel und Immunreaktivität stellen demzufolge eine Funktion der Zeit dar.

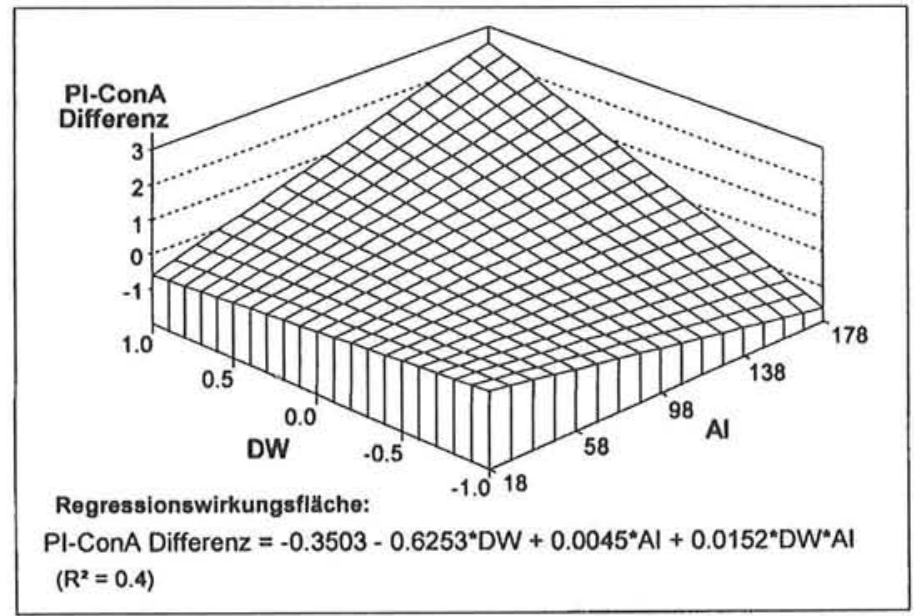

Abb. 2: Änderung der ConA-induzierten Lymphozytenproliferationsraten (PI) im Zeitraum zwischen einem Tag vor dem Mischen der Tiere und nach Herausbildung der Rangordnung in Abhängigkeit vom Dominanzwert (DW) und der Anzahl agonistischer Interaktionen (AI) (ConA stimulated proliferation index change between 1 day before mixing and after establishment of social rank order in relation to the dominance value and the number of agonistic interactions) (TUCHSCHERER et al., 1998))

In eigenen Untersuchungen führte das Mischen von 12 Wochen alten Schweinegruppen zu offenen Rangkämpfen, um eine neue soziale Hierarchiestruktur zu etablieren. Der Dominanzstatus beeinflußte die Lymphozytenproliferationsfähigkeit nach unterschiedlicher Mitogenstimulierung wesentlich: Dominante Tiere wiesen eine höhere Proliferationsfähigkeit als subdominante auf. Während der Herausbildung der Rangordnung erhöhte sich die zellvermittelte Immunreaktivität der dominanten Tiere mit steigender Anzahl gewonnener Kämpfe, die der subdominanten Tiere hingegen verrin- 
gerte sich mit höherer Zahl verlorener Rangkämpfe (Abb. 2). Die Ergebnisse verdeutlichen, daß akute psychosoziale Belastungen beim Schwein sowohl immunsuppressiv als auch immunstimulierend wirken können. Ob eine Hemmung oder eine Aktivierung immunologischer Funktionen auftritt, hängt davon $\mathrm{ab}$, wie diese Belastungen vom einzelnen Individuum bewältigt werden. Die T-zellvermittelte Immunreaktivität scheint besonders sensitiv auf akute soziale Belastungen zu reagieren (TUCHSCHERER et al., 1998).

Daß Effekte von Belastungssituationen auf Immunfunktionen nicht nur in vitro sondern auch in vivo nachweisbar sind, zeigen die Infektionsversuche mit Aujeszky Virus von HESSING et al. (1994). Dominante Schweine wiesen im Vergleich zu subordinaten und subdominanten Tieren eine höhere Resistenz gegenüber Aujeszky Virus auf, wobei unter den subordinaten Tieren die höchsten Morbiditäts- und Mortalitătsraten auftraten.

Absetzferkel, die in kleinere Buchten umgestallt und mit unbekannten Tieren zu neuen Gruppen zusammengestellt wurden, reagierten auf diese Belastungen im PHAHauttest verzögert und mit einer verringerten Induration (Ödembildung) im Vergleich zu Kontrolltieren (EKKEL et al., 1995). Ein geringeres Platzangebot pro Tier fuhrte bei Mastschweinen zu verringerter zellvermittelter Immunität (BARNETT et al., 1992).

Ebenfalls eine verringerte mitogeninduzierte Lymphozytenreaktivität und sinkende Plasmacortisolspiegel wurden nach Haltung von Schweinen bei konstant hohen Umgebungstemperaturen von $32^{\circ} \mathrm{C}$ im Vergleich zu bei $21^{\circ} \mathrm{C}$ gehaltenen Tieren nachgewiesen (BECKER und MISFELDT, 1995).

Das Absetzen vom Muttertier ist in der landwirtschaftlichen Nutztierhaltung eine Belastungssituation für die Nachkommen, an die sie sich möglichst schnell anpassen sollen. Signifikante Abnahmen der Lymphozytenzahl traten besonders bei den Ferkeln auf, bei denen sich das Haltungssystem und die Sozialzusammenstellung beim Absetzen änderten (TUCHSCHERER et al., 1994).

Die Laufbandbelastung ( $5 \mathrm{~min}$ ) von 3 Monate alten Schweinen führte zu einem sofortigen Anstieg der Plasmacortissolkonzentration, die bis 60 min nach dieser Bewegungsübung erhalten blieb. Weder die Transformationsfahhigkeit der Lymphozyten noch ihre Interleukin-2- bzw. Interferon- $\alpha$-Produktion waren jedoch bis $72 \mathrm{~h}$ nach Belastungsende beeinträchtigt (JENSEN WAERN und FOSSUM, 1993).

\section{$2.4 \quad$ Pferd}

Bewegungstraining verursachte bei jüngeren Pferden eine wesentliche Verminderung der T- und B-Lymphozytenproliferation, die Killerzellaktivität erhöhte sich jedoch leicht mit der Trainingsintensität. Bei älteren Pferden, die bereits vor dem Training eine niedrigere mitogeninduzierbare Lymphozytenproliferationsfähigkeit im Vergleich zu jüngeren Pferden besaßen, wurde diese durch das Training nicht negativ beeinflußt. Möglicherweise sind hierfür die geringeren Plasmacortisolspiegel bei älteren Tieren verantwortlich. Aufgrund der komplexen Interaktionen zwischen Hormon- und Immunsystem könnten auch andere Mediatoren, einschließlich Katecholamine, ACTH und CRH eine Rolle in der Immunmodulation spielen, da diese Mediatoren direkten Einfluß auf Pferdelymphozyten in vitro ausübten (HOROHOV et al., 1999). 


\subsection{Geflügel}

Akute soziale Isolation von Hühnerküken führte kurzzeitig zur Erhöhung der BZellproliferationsleistungen, induzierte jedoch bei längerer Dauer eine Verringerung der T-Zellproliferationsfähigkeit. Diese streßinduzierten Veränderungen der Immunreaktivität waren mit unterschiedlichen IL-1-Produktionraten von Milzmonozyten verbunden und unterstreichen die zentrale Bedeutung von IL-1 als Mediator innerhalb der psychoneuro-immunologischen Kommunikation (CUNNICK et al., 1994).

Bei Hühnerküken führten sowohl Hitze- als auch Kältebelastung zur Hemmung der zellvermittelten Immunität in vivo und in vitro (REGNIER und KELLY, 1981).

3. Schlußfolgerungen

Der generell negative Einfluß von (Di-) Streß auf die Immunfunktion kann inzwischen als gesichert angesehen werden (MAYR und MAYR, 1998). Wesentlich weniger klar ist beim gegenwärtigen Stand des Wissens, wie im einzelnen unterschiedliche Stressoren auf das Immunsystem einwirken. Daher ist es nicht möglich, genaue Voraussagen auf die Veränderung von immunologischen Funktionen in Folge einer spezifischen psychischen Streßbelastung zu treffen. Dies gilt insbesondere, da die Bewertung eines Reizes als Stressor eine Spezies- und Individualeigenschaft ist.

Aus verschiedenen Gründen wird in der zukünftigen landwirtschaftlichen Tierhaltung der Einsatz von Pharmaka verringert werden. Um dennoch gesunde und leistungsstarke Tiere zu haben, wird man die natürliche Krankheitsresistenz weitestgehend nutzen wollen und müssen. Insofern sind Arbeiten zur Immunkompetenz der Tiere unter verschiedenen Haltungsbedingungen grundlegend, um Distreßsituationen in zukünftigen Tierhaltungssystemen zu reduzieren und dadurch die natürliche Immunität zu verbessern. Schwerpunkt werden dabei der unspezifische Schutz auf zellulärer Ebene als sofort reagierender Teil der körpereigenen Abwehr gegen eingedrungene Krankheitskeime sein sowie die Aktivität von antigenpräsentierenden Monozyten/Makrophagen, die u.a. entscheidend für die Ausprägung der antigenspezifischen Immunität sind.

Außer Frage steht, daß ungeeignete bzw. belastende Haltungsbedingungen krankheitsfördernd sind (HESSING et al., 1994; FERRANTE et al., 1998). Zu beachten sind auch sogenannte latente Infektionen, die, trotz hygienisch einwandfreier Haltung, ausbrechen können, wenn die Streßbelastung ansteigt (GLASER et al., 1985). Insgesamt können erhöhte Krankheitsanfälligkeit sowie ein stark negativ veränderter Immunstatus der meisten Tiere einer Herde unter bestimmten Randbedingungen auf nicht tiergerechte Haltung hinweisen (MANTEUFFEL und PUPPE, 1997).

Zur Verbesserung der natürlichen Tiergesundheit sollten - neben den notwendigen hygienischen Maßnahmen - Haltungssysteme entwickelt werden, die nicht nur Distreß vermeiden, sondern zumindest zeitweise Eustreß ermöglichen. Aufgabe der Forschung ist es, zu ermitteln, was für eine gegebene Nutztierspezies di- und eustressend ist. Hinsichtlich der wahrscheinlich verteuerten Haltung müssen außerdem Lösungen gefunden werden, die die Haltungskosten gegenüber dem erhöhten betrieblichen Gewinn durch verminderten Pharmakaeinsatz und verbesserte Produktqualität optimieren und dabei auch den volkswirtschaftlichen und medizinischen Nutzen durch das Vermeiden antibiotikaresistenter Keime in die Betrachtung einbeziehen. 


\section{Literatur}

ADER, R.; COHEN, N.:

Behaviorally conditioned immunosuppression. Psychosom. Med., 37 (1975) 4, 333-340

ADER, R.; FELTEN, D.; COHEN, N.: Interaction between the brain and the immune system. Ann. Rev. Pharmacol. Toxicol., 30 (1990), 561-
602

ATHREYA, B.H.; PLETCHER, J.; ZULIAN, F.; WEINER, D.B.; WILLIAMS, W.V.; Subset-specific effects of sex hormones and pituitary gonadotropins on human lymphocyte proliferation in vitro. Clin. Immunol. Immunopathol., 66 (1993) 3, 93 BARNETT, J.L.; HEMSWORTH, P.H.; CRONIN, G.M.; NEWMAN, E.A.; McCALLUM, T.H., CHILTON,
D.: Effects of pen size, partial stalls and method of feeding on welfare-related behavioural and

physiological responses of group-housed pigs. Appl. Anim. Behav. Sci., Amsterdam 34 (1992), 207-220
BECKER, B.A.; MISFELDT, M.L.: Effects of constant and cycling hot environments on mitogen-stimulated proliferation of peripheral blood lymphocytes from sows and litters. J. therm. Biol., 20 (1995) 6, 485-488

BISWAS, R.; CHATTOPADHYAY, U.: Altered prolactin response of the lymphocytes of tumor-bearing mice. Int. J. Cancer., 50 (1992) 1, 93-98 BLALOCK, J.E.: The syntax of immune-neuroendocrine communication. Immunol. Today, 15 (1994) 11, 504-511

BLUTHÉ, R.M.; CRESTANI, F.; KELLY, K.W.; DANTZER, R.; Mechanisms of the behavioral effects of interleukin-1: Role of prostaglandins and CRF. Ann. N. Y.
Acad. Sci., 650 (1992), 268-275 BURCHFIELD, S.R. The stress response: A new perspective. Psychosom. Med., 41 (1979) 8, 661-672

CHROUSOS, G.P.; GOLD, P.W. The concepts of stress and stress system disorders. JAMA, 267 (1992), 1244-1252

COCKRAM, M.S.; RANSON, M.; IMLAH, P.; GODDARD, P. J.; BURRELLS, C.; HARKISS, G.D.: The behavioural, endocrine and immune responses of sheep to isolation. Anim. Prod., Edinburgh 58
(1994), 389-399

COMSA, J.; LEONHARDT, H.; WEKERLE, H.: Hormonal coordination of the immune response. Rev. Physiol. Biochem. Pharmacol., 92 (1982), 116-
191

CUNNICK, J.E.; KOJIC, L.D.; HUGHES, R.A.:

Stress-induced changes in immune function are associated with increased production of an interleukin1-like factor in young domestic fowl. Brain Behav. Immun., San Diego 8 (1994), 123-136

DALIN, A.-M.; MAGNUSSON, U.; HÄGGENDAL, J.; NYBERG, L.: The effect of transport stress on plasma levels of catecholamines, cortisol, corticosteroid-binding globulin, blood cell count, and lymphocyte proliferation in pigs. Acta vet. scand., 34 (1993) 1, 59-68

DANTZER, R.; BLUTHÉ, R.M.; KENT, S.; GOODALL, G.: Behavioral effects of cytokines: An insight into mechanisms of sickness behavior. In: DE SOUZA, E.

DEGUCHI, E.; AKUZAWA, M.: Effects of fighting after grouping on plasma cortisol concentration and lymphocyte blastogenesis of peripheral blood mononuclear cells induced by mitogens in piglets. J. Med. Sci., 60 (1998) 2, 149-153

EKKEL, E.D.; KUYPERS, A.H.; COUNOTTE, G.H.M.; TIELEN, M.J.M.: The phytohaemagglutinin (PHA) skin test as an indicator of stress-induced changes in immune FABRY, Z.; RAINE, C.S.; HART, M.N.: Nervous tissue as an immune compartment: the dialect of the immune response in the CNS. Immunol. Today, 15 (1994), 218-224

FELTEN, D.L.; FELTEN, S.; CARLSON, S.; OLSCHOWKA, J.; LIVNAT, S.: Noradrenergic and peptidergic innervation of lymphoid tissue. J. Immunol., 135 (1985), 755-765 FERRANTE, V.; CANALI, E.; MATTIELLO, S.; VERGA, M.; SACERDOTE, P.; MANFREDI, B.;
PANERAI, A.E.: Preliminary study on the effect of size of individual stall on the behavioural and immune reactions of dairy calves. J. Anim. Feed Sci., 7 (1998), 29-36 
GLASER, R.; KIECOLT-GLASER, J.K.; SPEICHER, C.E.; HOLLIDAY, J.:

Stress, loneliness, and changes in herpesvirus latency. J. Behav. Med., 8 (1985), 249-260

HESSING, M.J.C.; SCHEEPENS, C.J.M.; SCHOUTEN, W.G.P.; TIELEN, M.J.M.; WIEPKEMA, P.R.: Social rank and disease susceptibility in pigs. Vet. Immunol. Immunopathol., Amsterdam 43 (1994), 373-387

HICKS, T.A.; McGLONE, J.J.; WHISNANT, C.S.; KATTESH, H.G.; NORMAN, R.L.: Behavioral, endocrine, immune, and performance measures for pigs exposed to acute stress. J. Anim. Sci., Savoy 76 (1998), 474-483

HOROHOV, D.W.; DIMOCK, A.; GUIRNALDA, P.; FOLSOM, R.W.; McKEEVER, K.H.; MALINOWSKI,

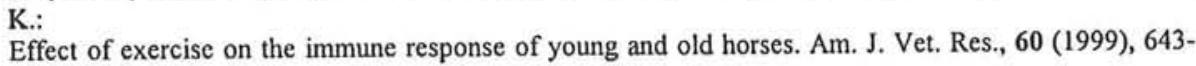
647

JENSEN WAERN, M; FOSSUM, C.: Effects of acute physical stress on immune competence in pigs. Am. J. Vet. Res., 54 (1993) 4, 596-601

JOHNSON, R.W.; VON BORELL, E.: Lipopolysaccharide-induced sickness behavior in pigs is inhibited by pretreatment with indomethacin. J. Anim. Sci., Savoy 72 (1994), 309-314

JOHNSON, R.W.; VON BORELL, E.; ANDERSON, L.L.; KOJIC, L.D.; CUNNICK, J.E.:

Intracerebroventricular infection of corticotropin-releasing hormone in the pig: Acute effects on behavior, adrenocorticotropin secretion, and immune suppression. Endocrinology, Baltimore 135 (1994) 2, 642-648

KARALIS, K.; MUGLIA, L.J.; BAE, D.; HILDERBRAND, H.; MAJZOUB, J.A.: CRH and the immune system. J. Neuroimmunol., 72 (1997), 131-136

KEGLEY, E.B.; SPEARS, J.W.; BROWN, T.T., Jr.: Effect of shipping and chromium supplementation on performance, immune response, and disease resistance of steers. J. Anim. Sci., Savoy 75 (1997), 1956-1964

KENT, S.; BLUTHÉ, R.M.; DANTZER, R.; HARDWICK, A.J.; KELLY, K.W.; ROTHWELL, N.J.; VANNICE, J.L.:

Different receptor mechanisms mediate the pyrogenic and behavioral effects of interleukin-1. Proc. Natl. Acad. Sci. USA, 89 (1992), 9117-9120

KIECOLT-GLASER, J.K.; GARNER, W.; SPEICHER, C.; PENN, G.M.; GLASER, R.:

Psychosocial modifiers of immunocompetence in medical students. Psychosomat. Med., 46 (1984), 714

KOLB, H.; REINHARDT, D.: Immunstimulation.Med. Welt, 41 (1990), 176-182

LAN, H.C.; REDDY, P.G.; CHAMBERS, M.A.; WALKER, G.; SRIVASTAVA, K.K.; FERGUSON, J.A.: Effect of stress on interleukin-2 receptor expression by bovine mononuclear leukocytes. Vet. Immunol. Immunopathol., Amsterdam 49 (1995), 241-249

LANDMANN, R.: Beta-adrenergic receptors in human leukocyte subpopulations. Eur. J. Clin. Invest., 22 (1992) Suppl. I, 30-36

LÖHRKE, B.; JENTSCH, W.; DERNO, M.; MATTHES, H.-D.: Widerspiegelung von Anpassungsvorgängen uber neuroendokrine und Immunreaktionen beim Rind. Arch. Tierz., Dummerstorf 37 (1994) 5, 493-508

MADDEN, K.S.; FELTEN, D.L.: Experimental basis for neural-immune interactions. Physiol. Rev., 75 (1995) 1, 77-106

MANTEUFFEL, G.; PUPPE, B.: Ist die Beurteilung der subjektiven Befindlichkeit von Tieren möglich? Eine kritische Analyse aus naturwissenschaftlicher Sicht. Arch. Tierz., Dummerstorf 40 (1997) 2, 109-121

MATERA, L.; CESANO, A.; BELLONE, G.; OBERHOLTZER, E.: Modulatory effect of prolactin on the resting and mitogen-induced activity of $T$, B, and NK lymphocytes. Brain Behav. Immun., San Diego 6 (1992) 4, 409-417

MAYR, B.; MAYR, A.: Interaktionen zwischen Immunsystem und Psyche. Tierärztl. Prax., 26 (1998), 230-235

McEWEN, B.S.; BIRON, C.A.; BRUNSON, K.W.; BULLOCH, K.; CHAMBERS, W.H.; DHABHAR, F.S.; GOLDFARB, R.H.; KITSON, R.P.; MILLER, A.H.; SPENCER, R.L.; WEISS, J.M.:

The role of adrenocorticoids as modulators of immune function in health and disease: Neural, endorcrine and immune interactions. Brain Res. Brain Res. Rev., 23 (1997) 1-2, 79-133 
MCGLONE, J.J.; SALAK, J.L.; LUMPKIN, E.A.; NICHOLSON, R.I.; GIBSON, M.; NORMAN, R.L.: Shipping stress and social status effects on pig performance, plasma cortisol, natural killer cell activity, and leukocyte numbers. J. Anim. Sci., Savoy 71 (1993), 888-896

MENCH, J.A.; SWANSON, J.C.; STRICKLIN, W.R.: Social stress and dominance among group members after mixing beef cows. Can. J. Anim. Sci., Ottawa 70 (1990) 2, 345-354

MINTON, J.E.; APPLE, J.K.; PARSONS, K.M.; BLECHA, F.:

Stress-associated concentrations of plasma cortisol cannot account for reduced lymphocyte function and changes in serum enzymes in lambs exposed to restraint and isolation stress. J. Anim. Sci., Savoy 73 (1995), 812-817

NIWANO, Y.; BECKER, B.A.; MITRA, R.; CALDWELL, C.W.; ABDALLA, E.B.; JOHNSON, H.D.: Suppressed peripheral blood lymphocyte blastogenesis in pre- and postpartal sheep by chronic heatstress, and suppressive property of heat-stressed sheep serum on lymphocytes. Dev. Comp. Immunol. 14 (1990), 139-149

ORGEUR, P.; MAVRIC, N.; YVORE, P.; BERNARD, S.; NOWAK, R.; SCHAAL, B.; LEVY, F.: Artificial weaning in sheep: consequences on behavioural, hormonal and immuno-pathological indicators of welfare. Appl. Anim. Behav. Sci., Amsterdam 58 (1998), 87-103

OTTAVIANI, E.; FRANCESCHI, C.: The neuroimmunology of stress from invertebrates to man. Progr. Neurobiol., 48 (1996), 421-440

PELLEGRINI, I.; LEBRUN, J.J.; ALI, S.; KELLY, P.A.: Expression of prolactin and its receptor in human lymphoid cells. Mol. Endocrinol. 6 (1992) 7, 1023 1031

REGNIER, J.A.; KELLEY, K.W.:

Heat- and cold-stress suppresses in vivo and in vitro cellular immune responses of chickens. Am. J. Vet. Res. 42 (1981) 2, 294-299

RHIND, S.M.; REID, H.W.; McMILLEN, S.R.; PALMARINI, G.:

The role of cortisol and $\beta$-endorphin in the response of the immune system to weaning in lambs. Anim.

RUNGE, J : Sci., Edinburgh 66 (1998), 397-402

Psychogen induzierte Stimulierung des Immunsystems. Diss. med. vet., München, 1991

SALAK-JOHNSON, J.L.; McGLONE, J.J.; NORMAN, R.L.: In vivo glucocorticoid effects on porcine natural killer cell activity and circulation leukocytes. J. Anim. Sci., Savoy 74 (1996), 584-592

SCHEDLOWSKI, M.; SCHMIDT, R.E.: Stress und Immunsystem. Naturwissenschaften,Heidelberg 83 (1996), 214-220

SCHLENKER, G.; LYHS, L.: Interaktionen zwischen dem zentralen Nervensystem und dem Immunsystem - Übersichtsreferat. Berl. Münch. Tierärztl. Wschr., Berlin, Hamburg 104 (1991) 7, 236-239

SCHOLE, J.: Physiologische Grundlagen der nichterreger- und nichtantigenspezifischen Abwehr. Der praktische Tierarzt, 63 (1982), 345-351

SELIGMAN, M.E.; BEAGLEY, G.:

SELYE, H.: Learned helplessness in the rat. J. Comp. Physiol. Psychol., 88 (1975) 2, 534-541

Stress and the reduction of distress. J. S. C. Med. Assoc., 75 (1979) 11, 562-566

STEFANO, G.B.: Role of opioid neuropeptides in immunregulation. Progr. Neurobiol., 33 (1989), 149-159

STEPHANOU, A.; JESSOP, D.S.; KNIGHT, R.A.; LIGHTMAN, S.L.: Corticotrophin-releasing factor-like immunoreactivity and mRNA in human leukocytes. Brain Behav. TEWES, U.: Immun., 4 (1990) 1, 67-73

Konzepte der Psychologie. In: SCHEDLOWSKI, M.; TEWES, U. (eds.): Psychoneuroimmunologie. Spektrum Akademischer Verlag, Heidelberg, 1996, 107-133

TUCHSCHERER, M.; PUPPE, B.; TUCHSCHERER, A.; TOBER, O.:

Verănderte Umwelt für Ferkel nach dem Absetzen von der Mutter - physiologische und ethologische Konsequenzen. Arch. Tierz., Dummerstorf 37 (1994) Sonderheft, 167

TUCHSCHERER, M.; PUPPE, B.; TUCHSCHERER, A.; KANITZ, E.:

Effects of social status after mixing on immune, metabolic, and endocrine responses in pigs. Physiol. Behav., Tarrytown 64 (1998) 3, 353-360 
VON HOLST, D.:

The concept of stress and its relevance for animal behavior. Adv. Study Behav., 27 (1998), 1-131

WARREN, E.J.; FINCK, B.N.; ARKINS, S.; KELLEY, K.W.; SCAMURRA, R.W.; MURTAUGH, M.P.; JOHNSON, R.W.:

Coincidental changes in behavior and plasma cortisol in unrestrained pigs after intracerebroventricular injection of tumor necrosis factor- $\alpha$. Endocrinology, Baltimore 138 (1997) 6, 2365-2371

WEBSTER, E.L.; ELENKOV, I.J.; CHROUSOS, G.P.:

The role of corticotropin-releasing hormone in neuroendocrine-immune interactions. Mol. Psychiatry, 2 (1997), 368-372

WEIGENT, D.A.; BLALOCK, J.E.:

Associations between the neuroendocrine and immune systems. J. Leukoc. Biol.., 57 (1995), 137-150

YANG, W.C.; SCHULTZ, R.D.:

Effect of corticosteroid on porcine leukocytes: Age-related effects of corticosteroid inhibition on porcine lymphocyte responses to mitogens. Vet. Immunol. Immunopathol., Amsterdam 13 (1986), 1929

Eingegangen: 09.02 .2000

Akzeptiert: 28.09 .2000

Anschrift der Verfasser

Dr. MARGRET TUCHSCHERER, Prof. Dr. habil. GERHARD MANTEUFFEL

Forschungsinstitut furr die Biologie landwirtschaftlicher Nutztiere (FBN)

Wilhelm-Stahl-Allee 2

D-18196 Dummerstorf

E-Mail: mtuchsch@fon-dummerstorf.de 